\title{
Dealing with delicate issues in waveforms calculations
}

\author{
Luis Lehner ${ }^{1}$ and Osvaldo M. Moreschi ${ }^{2}$ \\ ${ }^{1}$ Department of Physics \& Astronomy, LSU, \\ 16224 Baton Rouge, LA 70810. \\ ${ }^{2}$ FaMAF, Universidad Nacional de Córdoba \\ Ciudad Universitaria, (5000) Córdoba, Argentina.
}

\begin{abstract}
We revisit the calculation of gravitational radiation through the use of Weyl scalars. We point out several possible problems arising from gauge and tetrad ambiguities and ways to address them. Our analysis indicates how, relatively simple corrections can be introduced to remove these ambiguities.
\end{abstract}

PACS numbers:

\section{INTRODUCTION}

The definition of gravitational radiation involves a fair amount of work so as to distinguish variations in the metric that are purely coordinate effects from true signals propagating on a background which itself must be well established. At finite (but sufficiently far) distances from an isolated source several approaches exist to do so provided a suitable background can be identified [1, 2, 3, 4, 5]. For sufficiently generic cases the unambiguous identification of the gravitational radiation can only be done at future null infinity $\left(\mathcal{I}^{+}\right)$. This is possible since when studying an isolated system, future null infinity is the asymptotic region of an asymptotically flat spacetime. There the metric is exactly flat and disturbances around it can be associated with outgoing gravitational waves subject to singling out a preferred frame. This issue arises as there is no unique flat asymptotic metric. In fact there are as many of them as supertranslations are in the BMS group; the asymptotic symmetry group. Nevertheless there is a well defined notion of gravitational radiation which exploits the algebraic notion of asymptotic flatness [6] which in turn implies the existence of the gravitational radiation fields $\Psi_{4}^{0}$ and $\Psi_{3}^{0}$. The existence of these fields can also be deduced from stronger conditions, like peeling [7, 8]. Then, after a careful choice of coordinates, radiation can be unambiguously defined.

Unfortunately, numerical applications dealing with black hole spacetimes can not yet reach $\mathcal{I}^{+}$unless Cauchy-characteristic extraction is employed [9, 10] for the extraction procedure or Cauchy-characteristic matching [11] or the conformal formulations 12] are fully realized to model the whole spacetime. Without adopting any of these options a commonly employed approach for obtaining gravitational waves relies on applying the infrastructure developed for $\mathcal{I}^{+}$at finite distances. Here, several compromises are made as natural quantities defined at $\mathcal{I}^{+}$need to be translated somehow to finite distances where they need not be well defined.

The purpose of this note is to point out difficulties that likely arise in this approach and a route to address them.

\section{REVIEW OF THE APPROACH}

The calculation of gravitational radiation at $\mathcal{I}^{+}$is based on the foundational works of Bondi [13] and Sachs [14] as an approximation expansion at infinite distances and by the work of Penrose [15] as a geometrical construct at the boundary of the physical spacetime. Under reasonable assumptions one can show that the spacetime is asymptotically flat and time dependent perturbations close to the boundary are either due to gravitational waves or coordinate effects. In order to disentangle coordinate with physical effects, care must be taken in dealing with the asymptotic symmetry group and fixing a convenient frame with respect to which inertial observers can define radiation.

Our goal here is not to revisit this approach (of which descriptions have been presented in e.g. 8, 16, 17, 18, 19, 20]), but simply to state the crucial ingredients which are missing when the strategy is employed at finite distances. In the next sections we will carry out an analysis of what corrections are required when key assumptions described below are missing.

These ingredients are,

- Peeling is assumed, this means that suitable Weyl curvature components behave asymptotically in a well defined manner.

- Outgoing null hypersurfaces, parameterized by $u$ intersect $\mathcal{I}^{+}$defining a sequence of $S^{2}$ surfaces.

- Each of these surfaces is conformal to a unit sphere metric; off this surface (into the spacetime) the departure from it is of lower order. Namely the angular metric in a neighborhood of $\mathcal{I}^{+}$can be expressed, as $g_{A B}=r^{2} h_{A B}=r^{2}\left(q_{A B} / V^{2}+c_{A B} / r+\right.$ $\left.O\left(r^{-2}\right)\right)$; with $V$ a conformal factor, $q_{A B}$ a unit sphere metric, and $r$ a suitably defined radial distance.

- A null-tetrad $\left\{\ell^{a}, n^{a}, m^{a}, \bar{m}^{a}\right\}$ satisfying $\ell^{a} n_{a}=$ $-m^{a} \bar{m}_{a}=1$ (with all other products being 0). The spacetime metric can be expressed as $g_{a b}=$ $2 l_{(a} n_{b)}-2 m_{(a} \bar{m}_{b)}$, and suitable projections of the Weyl tensor can be defined. 
- Using standard 21, 22] conventions for the Riemann tensor and spinor dyad, particularly useful scalars obtained from them are,

$$
\begin{aligned}
\Psi_{4} & =C_{a b c d} n^{a} \bar{m}^{b} n^{c} \bar{m}^{d} \\
\Psi_{3} & =C_{a b c d} \ell^{a} n^{b} \bar{m}^{c} \bar{n}^{d} \\
\Psi_{2} & =C_{a b c d} \ell^{a} m^{b} \bar{m}^{c} n^{d} \\
\sigma & =m^{a} m^{b} \nabla_{a} l_{b} .
\end{aligned}
$$

- A suitable (Bondi type) expansion in terms of $1 / r$ coupled to coordinates chosen such that $V=1$, $g_{u r}^{0}=1$ and $g_{u A}^{0}=0\left(x^{A}\right.$ labeling angular coordinates at $u=$ const, $r \rightarrow \infty)$ gives rise to several important relations [6, 8]. In particular,

$$
\begin{aligned}
\Psi_{4}^{0} & =-\ddot{\bar{\sigma}}^{0} \\
\Psi_{3}^{0} & =-\partial \dot{\bar{\sigma}}^{0} \\
\dot{\Psi}_{2}^{0} & =\partial \Psi_{3}^{0}+\sigma^{0} \Psi_{4}^{0} ;
\end{aligned}
$$

where the supra-index "0" indicates leading order in an expansion in the radial coordinate and $\partial$ is the edth operator 21] of the unit sphere.

With this structure at hand, the following results are obtained. Given any section $S$ at future null infinity, the Bondi momentum is given by

$$
P^{a}=-\frac{1}{4 \pi} \int_{S} \hat{l}^{a}\left(\Psi_{2}^{0}+\sigma^{0} \dot{\bar{\sigma}}^{0}\right) d S^{2},
$$

where $\hat{l}^{a}=(1, \sin (\theta) \cos (\phi), \sin (\theta) \sin (\phi), \cos (\theta))$ when expressed in standard angular coordinates $(\theta, \phi)$. The so called Bondi mass $M$ is the timelike component of this vector; namely

$$
M=-\frac{1}{4 \pi} \int_{S}\left(\Psi_{2}^{0}+\sigma^{0} \dot{\bar{\sigma}}^{0}\right) d S^{2} .
$$

It is interesting to note that the Bondi momentum can also be expressed in the terms of the Psi supermomentum [23] $\Psi \equiv \Psi_{2}^{0}+\sigma^{0} \dot{\bar{\sigma}}^{0}+\check{\partial}^{2} \bar{\sigma}^{0}$ by

$$
P^{a}=-\frac{1}{4 \pi} \int_{S} \hat{l}^{a} \Psi d S^{2} ;
$$

since it has a couple of useful properties; namely, it is real, $\bar{\Psi}=\Psi$, and its time derivative is simply

$$
\dot{\Psi}=\dot{\sigma}^{0} \dot{\bar{\sigma}}^{0}
$$

Therefore, the time variation of the Bondi momentum, in terms of time Bondi coordinate, is just given by

$$
\dot{P}^{a}=-\frac{1}{4 \pi} \int_{S} \hat{l}^{a} \dot{\sigma}^{0} \dot{\bar{\sigma}}^{0} d S^{2} .
$$

Notice that due to relation (5) one might choose to replace any appearance of $\dot{\sigma}^{0}$ by the time integral of $\Psi_{4}^{0}$. This is commonly done in numerical simulations [24, 25, 26, 27, 28, 29, 30, 31, 32, 33]; however one should notice that (5) is only valid in terms of a Bondi tetrad and coordinate system.

\section{CONNECTION WITH FINITE DISTANCES}

The above expressions provide a well defined infrastructure with which gravitational radiation can be defined. However, to obtain them one has introduced key ingredients, otherwise the expressions would result significantly more involved. In particular without choosing the conformal frame and/or radial distance such that the leading part of the angular metric is indeed that of the unit sphere, several changes arise even when all other properties are satisfied. For instance, if the angular metric were given by $g_{A B}=r^{2}\left(q_{A B} / V(u, \theta, \phi)^{2}+O\left(r^{-1}\right)\right)$ then, instead of (5) one would have

$$
\begin{aligned}
& \Psi_{4}^{0}=-\ddot{\bar{\sigma}}^{0}-\frac{1}{V} \bar{\partial}^{2} \dot{V}+\frac{2}{V^{2}} \overline{\bar{\partial}} \dot{V} \bar{\varnothing} V-\frac{2}{V^{3}} \dot{V}(\bar{\varnothing} V)^{2} \\
& +\frac{1}{V^{2}} \dot{V} \bar{\partial}^{2} V-\frac{3}{V^{2}} \dot{V}^{2} \bar{\sigma}^{0}+\frac{1}{V} \ddot{V} \bar{\sigma}^{0}+\frac{3}{V} \dot{V} \dot{\bar{\sigma}}^{0} \\
& =-\ddot{\bar{\sigma}}^{0}-\overline{\bar{\delta}}^{2} \frac{\dot{V}}{V}-\frac{3}{V^{2}} \dot{V}^{2} \bar{\sigma}^{0}+\frac{1}{V} \ddot{V} \bar{\sigma}^{0}+\frac{3}{V} \dot{V} \dot{\bar{\sigma}}^{0} .
\end{aligned}
$$

Therefore, if we call $\tilde{\Psi}_{4}^{0}$ the inertial (Bondi) radiation field one would have the relation

$\tilde{\Psi}_{4}^{0}=\frac{1}{V^{3}}\left(-\ddot{\bar{\sigma}}^{0}-\overline{\mathrm{\partial}}^{2} \frac{\dot{V}}{V}-\frac{3}{V^{2}} \dot{V}^{2} \bar{\sigma}^{0}+\frac{1}{V} \ddot{V} \bar{\sigma}^{0}+\frac{3}{V} \dot{V} \dot{\bar{\sigma}}^{0}\right)$.

where the factor $V^{-3}$ results from one of the corrections which we discuss in this work. Notice that this is not a perverse circumstance, rather it can be generically expected as surfaces at $u=$ const, $r=$ const define a 2 sphere whose metric is always conformally related to the unit sphere.

Certainly, in principle coordinate conditions can be adopted so that at the extraction sphere the simplifying conditions hold, these conditions need not be those that are convenient for the numerical implementations. Indeed, coordinate conditions are exploited in a non-trivial manner to aid in the numerical simulation 34, 35, 36. Therefore, one is then left with having to consider how to correctly identify suitable coordinates and/or a frame to extract the desired quantity. Here, one of two options can be adopted. One approach is motivated from a perturbative point of view and relies in extracting a suitable Kinnersley tetrad 37] from the numerically generated spacetime 38, 39, 40]. The so called Kinnersley tetrads are null tetrads that were constructed for the study of type $\mathrm{D}$ vacuum spacetimes; but one can in general choose them so that to leading order they conform to a Bondi tetrad. The basic idea is to use a null tetrad adapted to the asymptotic structure so that in the limit coincides with a Bondi tetrad. Armed with this tetrad the computation of the radiative properties of the spacetime is then well defined. The delicate point however stems from the fact that this approach requires a suitable numerical identification of a Bondi frame where errors can arise. The influence of these errors in a related context has been recently discussed in 41]. 
A different approach is to consider the asymptotic structure of asymptotically flat spacetimes and define the analog quantities at finite distances. This approach, which we adopt here, does not rely on obtaining background quantities correctly, rather one needs only identify a suitable Bondi frame which will essentially be unambiguous as long as the extraction worldtube is sufficiently far. We will not concern ourselves with how far must the extraction worldtube be, rather we assume one is able to place it sufficiently far for the peeling property, in the extracted quantities, of the Weyl scalars to be observed. Under this assumption we analyze what consequences, and most importantly, modifications one must take into account when calculating physical observables with the coordinates naturally induced on the extraction worldtube in a numerical simulation.

To this end, it will be important to calculate the induced metric on the extraction worldtube and evaluate different quantities which play a key role. We begin by following precisely the same procedure commonly employed in numerical simulations (see for instance 24, 28, 42]). First a Cartesian extraction worldtube is defined by $x^{2}+y^{2}+z^{2}=R^{2}$. Observers at this worldtube are defined by their trajectories on the worldtube given by ( $t, x=$ const., $y=$ const., $z=$ const.). Then, $\Psi_{4}$ is calculated on the hypersurface $\Sigma_{t}$ (defined by $t=$ const.) by suitably adopting a tetrad frame. This tetrad is chosen in a straightforward manner by adopting three orthonormal spatial vectors $\left\{r^{a}, \theta^{a}, \phi^{a}\right\}$ on $\Sigma_{t}$ (which are the analogs to the radial and tangents vectors to the sphere) and combine them with the unit normal vector $N^{a}$ to the hypersurface. Once $\Psi_{4}$ is obtained, an interpolation on to the extraction sphere is carried out. At this point it is useful to recall that the extraction must be done along null rays, thus any comparison (or refinements of the extracted quantities [49]) at different worldtubes should contemplate suitable time offsets. This offset reflects the arrival time of a null signal from a given internal worldtube to the next.

As mentioned, this procedure, need not give rise to a structure fully compatible with those mentioned in section I. In particular the induced angular sphere metric need not be sufficiently close to that of the unit sphere, inertial observers coordinates might 'shift' in time around the worldtube and their associated times might tick at different rates. At finite distances these issues are even more relevant since the waves themselves will influence the geometry of the extraction worldtube as they propagate across it. In the next section we discuss the corrections required and will illustrate their application in section V. As we will see, even in linearized problems these issues can play a non-trivial role.

A short summary of what these corrections entail is (in addition to the standard first-step to calculate $\Psi_{4}$ ) is,

- Assuming that the extraction world tube has been chosen far enough [50], calculate the leading order behavior $\Psi_{4}^{0}$ from $\Psi_{4}=\Psi_{4}^{0} r^{-1}+O\left(r^{-2}\right)$; where $r$ is an appropriate asymptotic radial coordinate; such that $r=R$ at the extraction world tube.

- In a similar way, obtain the leading order behavior of the induced metric $g_{\Gamma}$ on the sphere at the extraction worldtube defined by $t=$ const., $r=R$, from $g_{\Gamma}=-q R^{2} V^{-2}+O\left(r^{1}\right)$; where $q$ is the unit sphere metric.

- Obtain the leading order behavior $g_{u r}^{0}$ from the expansion $g_{u r}=g_{u r}^{0}+O\left(r^{-1}\right)$; which measures the time observers of $\Psi_{4}$ have.

- Obtain the leading order behavior $g_{u A}^{0}$ from the expansion $g_{u A}=g_{u A}^{0}+O\left(r^{-1}\right)$; which measures the coordinate shift in time observers of $\Psi_{4}$ have around the worldtube.

- Bondi's radiation $\tilde{\Psi}_{4}^{0}$ is then obtained by relatively simple correction factors from the knowledge of $\left\{g_{u r}^{0}, g_{u A}^{0}, V\right\}$. In the particular case where $g_{u A}^{0} \simeq 0$ the expression is

$$
\tilde{\Psi}_{4}^{0}=\frac{1}{\left(g_{u r}^{0}\right)^{2} V^{3}} \Psi_{4}^{0}
$$

\section{ASYMPTOTIC STRUCTURE OF ASYMPTOTICALLY FLAT SPACETIMES}

In this section we examine which corrections must be taken into account to remove the ambiguities described previously. To this end, we will examine how the relevant quantities transform among different coordinate systems compatible with the asymptotic structure. Then, by adopting one of these systems to be a Bondi one, we will be able to extract the correcting terms. For the sake of simplicity in the derivation we adopt the spinor-calculus approach, though the results obtained are completely independent of this technique.

We begin by determining key relations between different frames, to do so it is convenient to have at hand some basic equations of the asymptotic structure.

\section{A. Basic variables}

The asymptotic geometry can be expressed in terms of a complex null tetrad $\left(\ell^{a}, m^{a}, \bar{m}^{a}, n^{a}\right)$ with the properties:

$$
g_{a b} \ell^{a} n^{b}=-g_{a b} m^{a} \bar{m}^{b}=1
$$

and all other possible scalar products being zero, the metric can be expressed by

$$
g_{a b}=\ell_{a} n_{b}+n_{a} \ell_{b}-m_{a} \bar{m}_{b}-\bar{m}_{a} m_{b} .
$$

Such a null tetrad is easily related to a dyad of spinors $\left(o^{A}, \iota^{A}\right)$. The relation of the null tetrad with a spinor 
dyad is given by $\ell^{a} \Longleftrightarrow o^{A} O^{A^{\prime}}, m^{a} \Longleftrightarrow o^{A} \iota^{A^{\prime}}$, $\bar{m}^{a} \Longleftrightarrow \iota^{A} O^{A^{\prime}}$ and $n^{a} \Longleftrightarrow \iota^{A} \iota^{A^{\prime}}$. Thus, determining how the tetrad trasnforms one infers the transformation of $\left(o^{A}, \iota^{A}\right)$. In our discussion, to make a direct contact with the standard literature on the subject we also adopt a null polar coordinate system $\left(x^{0}, x^{1}, x^{2}, x^{3}\right)=$ $\left(u, r,(\zeta+\bar{\zeta}), \frac{1}{i}(\zeta-\bar{\zeta})\right)$. However, as opposed to the standard discussions we will not make use of the available coordinate freedom to simplify the treatment at the onset. Rather, we will adopt coordinates consistent with those employed in the extraction procedure at finite distances and deal with the consequences of this choice.

In most numerical applications, one normally works with finite size regions; and would like to estimate the asymptotic fields in terms of null tetrads based on a choice of coordinate system. At the extraction worldtube, one assumes that a coordinate system $(t, r, \zeta, \bar{\zeta})$ can be constructed. Let $\Gamma$, be the timelike surface defined by ( $r=R=$ const.). On $\Gamma$, a null tetrad $\ell, n, m, \bar{m}$ can be defined in the following way. First, define the null function $u$ such that on $\Gamma$ one has $u=t$; and $\ell=d u$ everywhere. The function $u$ is chosen such that the future directed vector $\ell$ points outwards with respect to the 2 -surfaces ( $t=$ const., $r=R)$; which are, topologically, two dimensional spheres.

The complex vectors $m$ and $\bar{m}$ are defined to be tangent to the spheres ( $t=$ const., $r=$ const.). Furthermore, one can choose $m$ to be proportional to $\frac{\partial}{\partial \zeta}$; and $\bar{m}$ to be proportional to $\frac{\partial}{\partial \zeta}$ in the asymptotic region for large $r$.

Then, by requiring the standard normalization conditions given in (16) one settles the remaining null vector $n^{a}$.

The coordinate system $(u, r, \zeta, \bar{\zeta})$ is thus straightforwardly related to the one commonly employed in numerical efforts $(t, R, \theta, \phi)$ though we will continue our discussion with the former as it is the one employed in the standard literature on the subject. The conclusion however, will be independent of this choice.

Keeping ( $\zeta=$ const.) and $(\bar{\zeta}=$ const. $)$ on the null hypersurface $u=$ const., and increasing $r$ one moves along a null direction. Since $\ell$ is contained on the hypersurface $u=$ const., one deduces that $\ell$ is proportional to $\frac{\partial}{\partial r}$. Then, one can write

$$
\left(\ell^{a}\right)=\left(\frac{1}{g_{u r}} \frac{\partial}{\partial r}\right)^{a}
$$

The appearance of $g_{u r}$ in the denominator is the first correction to be accounted for due to the structure induced at $\Gamma$ not necessarily conforming to a Bondi frame. The intersection of the null hypersurface $u=$ const. with future null infinity defines a two dimensional surface denoted by $S$. A natural null tetrad is then completed with,

$$
\begin{gathered}
\ell_{a}=(d u)_{a} \\
m^{a}=\xi^{i}\left(\frac{\partial}{\partial x^{i}}\right)^{a}
\end{gathered}
$$

$$
\begin{gathered}
\bar{m}^{a}=\bar{\xi}^{i}\left(\frac{\partial}{\partial x^{i}}\right)^{a} \\
n^{a}=\left(\frac{\partial}{\partial u}\right)^{a}+U\left(\frac{\partial}{\partial r}\right)^{a}+X^{i}\left(\frac{\partial}{\partial x^{i}}\right)^{a}
\end{gathered}
$$

with $i=2,3$ and components $\xi^{i}, U$ and $X^{i}$ are:

$$
\xi^{2}=\frac{\xi_{0}^{2}}{r}+O\left(\frac{1}{r^{2}}\right), \quad \xi^{3}=\frac{\xi_{0}^{3}}{r}+O\left(\frac{1}{r^{2}}\right),
$$

with

$$
\xi_{0}^{2}=\sqrt{2} P_{0} V, \quad \xi_{0}^{3}=-i \xi_{0}^{2}
$$

where $V=V(u, \zeta, \bar{\zeta})$ and the square of $P_{0}=\frac{(1+\zeta \bar{\zeta})}{2}$ is the conformal factor of the unit sphere;

$$
U=r U_{00}+U_{0}+\frac{U_{1}}{r}+O\left(\frac{1}{r^{2}}\right),
$$

where

$$
U_{00}=\frac{\dot{V}}{V}, \quad U_{0}=-\frac{1}{2} K_{V}, \quad U_{1}=-\frac{\Psi_{2}^{0}+\bar{\Psi}_{2}^{0}}{2},
$$

where $K_{V}$ is the Gaussian curvature, given by

$$
\begin{aligned}
K_{V} & =2 V \overline{\bar{\partial}} V-2 ð V \overline{\mathrm{\partial}} V+V^{2} \\
& =V \nabla^{2} V-\nabla^{i} V \nabla_{i} V+V^{2} \\
& =V^{2} \nabla^{2} \ln V+V^{2}
\end{aligned}
$$

of the 2-metric

$$
d S^{2}=\frac{1}{V^{2} P_{0}^{2}} d \zeta d \bar{\zeta}
$$

where the regular conformal metric restricted to $S$ is precisely $\left.\tilde{g}\right|_{S}=-d S^{2}, \nabla^{2}=\nabla^{i} \nabla_{i}$ is the Laplacian operator of the unit sphere metric and $\nabla_{i}$ its covariant derivative. Let us emphasize that $V=1$ makes $d S^{2}$ in (28) the unit sphere metric. Finally, the other components of the vector $n^{a}$ have the asymptotic form

$$
X^{2}=X_{2}^{0}+O\left(\frac{1}{r^{2}}\right), \quad X^{3}=X_{3}^{0}+O\left(\frac{1}{r^{2}}\right) .
$$

\section{B. Asymptotic gauge freedom, restricted case}

At this point, we find it convenient to restrict to a simplified case before describing the general one. We will assume here that $X_{2}^{0}=X_{3}^{0}=0$ which, in an alternative view implies that angular coordinates at $\Gamma$ do not shift in time as $g_{u A}^{0}=0$. Our discussion will center around the allowed transformations between the induced coordinates at the worldtube $(u, r, \zeta, \bar{\zeta})$ and the Bondi system $(\tilde{u}, \tilde{r}, \tilde{\zeta}, \overline{\tilde{\zeta}})$. 


\section{Coordinate and tetrad transformations $I$}

Let us consider the main gauge freedom admitted in our calculation which is of the form

$$
\begin{aligned}
& \tilde{u}=\alpha(u, \zeta, \bar{\zeta})+\frac{\tilde{u}_{1}(u, \zeta, \bar{\zeta})}{r}+O\left(\frac{1}{r^{2}}\right), \\
& \tilde{r}=\frac{r}{w(u, \zeta, \bar{\zeta})}+O\left(r^{0}\right), \\
& \tilde{\zeta}=\zeta+O\left(\frac{1}{r}\right) .
\end{aligned}
$$

with $\dot{\alpha}>0$. The possible further transformation of the coordinates of the sphere $(\zeta, \bar{\zeta})$ into itself is not needed at this point.

The condition $g^{\tilde{u} \tilde{r}}=1$ in a Bondi system imposes the relation

$$
w=\frac{\dot{\alpha}}{g_{u r}^{0}} .
$$

This asymptotic coordinate transformation is associated to a corresponding null tetrad transformation; which to leading orders is given by

$$
\begin{gathered}
\tilde{\ell}=d \tilde{u}=\dot{\alpha} d u+\alpha_{\zeta} d \zeta+\alpha_{\bar{\zeta}} d \bar{\zeta}+O\left(\frac{1}{r}\right) \\
=\dot{\alpha} \ell-\frac{\partial_{V} \alpha}{r} \bar{m}-\frac{\bar{\partial}_{V} \alpha}{r} m+O\left(\frac{1}{r}\right), \\
\tilde{n}=\frac{\partial}{\partial \tilde{u}}+O\left(\frac{1}{r}\right)=\frac{1}{\dot{\alpha}} \frac{\partial}{\partial u}+O\left(\frac{1}{r}\right) \\
=\frac{1}{\dot{\alpha}} n+O\left(\frac{1}{r}\right), \\
\tilde{m}=\frac{\sqrt{2} \tilde{P}}{\tilde{r}} \frac{\partial}{\partial \tilde{\zeta}}+O\left(\frac{1}{r^{2}}\right) \\
=\frac{\sqrt{2} P_{0} \tilde{V} w}{r}\left(-\frac{\alpha_{\zeta}}{\dot{\alpha}} \frac{\partial}{\partial u}+\frac{\partial}{\partial \zeta}\right)+O\left(\frac{1}{r^{2}}\right) \\
=-\frac{\sqrt{2} P_{0} \tilde{V} w}{r} \frac{\alpha_{\zeta}}{\dot{\alpha}} n+\frac{\tilde{V} w}{V} m+O\left(\frac{1}{r^{2}}\right)
\end{gathered}
$$

since the metric expressed in terms of the new null tetrad must coincide with the metric expressed in terms of the original null tetrad, it is deduced that

$$
\tilde{V}=\frac{V}{w}=\frac{V g_{u r}^{0}}{\dot{\alpha}} ;
$$

therefore

$$
\tilde{m}=m-\frac{{\mathrm{\partial}_{V}} \alpha}{r \dot{\alpha}} n+O\left(\frac{1}{r^{2}}\right) .
$$

The null tetrad transformation equations can be used to write the leading order transformation relations for the spinor dyad associated to the null tetrad 20, 21]; namely

$$
\tilde{o}^{A}=\sqrt{\dot{\alpha}}\left(o^{A}-\frac{\check{\partial}_{V} \alpha}{r \dot{\alpha}} \iota^{A}\right)
$$

and

$$
\tilde{\iota}^{A}=\frac{1}{\sqrt{\dot{\alpha}}} \iota^{A} ;
$$

where $\check{\partial}_{V}$ is the edth operator of the metric (28). Taking into account higher order transformations would include an equation of the form

$$
\tilde{\iota}^{A}=\frac{1}{\sqrt{\dot{\alpha}}}\left(\iota^{A}+h o^{A}\right) ;
$$

where in principle $h$ could be of order $O\left(r^{0}\right)$.

The regular dyad at future null infinity in terms of the spacetime one, can be given by

$$
\begin{gathered}
\hat{o}^{A}=\Omega^{-1} o^{A}, \\
\hat{\iota}^{A}=\iota^{A} .
\end{gathered}
$$

Then, the transformed regular dyad at future null infinity is given by

$$
\begin{gathered}
\hat{\tilde{o}}^{A}=\tilde{\Omega}^{-1} \tilde{o}^{A}=\frac{r}{w} \sqrt{\dot{\alpha}}\left(o^{A}-\frac{\partial_{V} \alpha}{r \dot{\alpha}} \iota^{A}\right) \\
=\frac{1}{\sqrt{\dot{\alpha}}}\left(\hat{o}^{A}-\frac{\partial_{V} \alpha}{\dot{\alpha}} \hat{\iota}^{A}\right), \\
\hat{\tilde{\iota}}^{A}=\tilde{\iota}^{A}=\frac{1}{\sqrt{\dot{\alpha}}} \hat{\iota}^{A} ;
\end{gathered}
$$

where we are using $\Omega=\frac{1}{r}$.

\section{Transformation of $\Psi_{4}^{0}$}

We can now easily calculate the component $\Psi_{4}$ of the Weyl tensor, in leading orders, with respect to the new null tetrad, obtaining

$$
\begin{aligned}
\tilde{\Psi}_{4}^{0} & =\tilde{\Omega}^{-1} \Psi_{A B C D} \hat{\tilde{\iota}}^{A} \hat{\tilde{\iota}}^{B} \hat{\tilde{\iota}}^{C} \hat{\tilde{\iota}}^{D}=\frac{1}{w \dot{\alpha}^{2}} \Psi_{4}^{0} \\
& =\frac{g_{u r}^{0}}{\dot{\alpha}^{3}} \Psi_{4}^{0}
\end{aligned}
$$

\section{Transformations to a Bondi system}

Having analyzed how to transform among different frames we can now consider our main task, to relate $\Psi_{4}$ calculated in an arbitrary frame to that which would be obtained in a Bondi frame. This frame satisfies, $g^{\tilde{u} \tilde{r}}=1$ and $\tilde{V}=1$ which implies

$$
\dot{\alpha}=V g_{u r}^{0} ; \dot{\alpha}=w g_{u r}^{0}
$$

Which fix both $w$ and $\dot{\alpha}$ and indicates how different quantities are to transform. In particular,

$$
\tilde{\Psi}_{4}^{0}=\frac{1}{V^{3}\left(g_{u r}^{0}\right)^{2}} \Psi_{4}^{0},
$$

Thus, knowledge of both $g_{u r}^{0}$ and $V$ allows one to obtain $\tilde{\Psi}_{4}$ in terms of the more directly calculated $\Psi_{4}$. We defer to section VI the discussion of how to obtain $g_{u r}^{0}$ and $V$. 


\section{Coordinate and tetrad transformations}

After examining the simpler case, we concentrate now on the general case, namely we will include the possibility of $X_{0}^{A} \neq 0$. As before, the task at hand is to transform to an asymptotic Bondi coordinate $(\tilde{u}, \tilde{r}, \tilde{\zeta}, \overline{\tilde{\zeta}})$ and tetrad frame $(\tilde{\ell}, \tilde{n}, \tilde{m}, \overline{\tilde{m}})$. This transformation is of the form

$$
\begin{aligned}
& \tilde{u}=\alpha(u, \zeta, \bar{\zeta})+\frac{\tilde{u}_{1}(u, \zeta, \bar{\zeta})}{r}+O\left(\frac{1}{r^{2}}\right), \\
& \tilde{r}=\frac{r}{w(u, \zeta, \bar{\zeta})}+O\left(r^{0}\right), \\
& \tilde{\zeta}=\tilde{\zeta}_{0}(u, \zeta, \bar{\zeta})+O\left(\frac{1}{r}\right) .
\end{aligned}
$$

with $\dot{\alpha}>0$.

If one assumes that $\zeta$ is an stereographic coordinate of the conformal unit sphere ( $t=$ const., $r=$ const.) it is only necessary to consider an angular transformation of the form

$$
\tilde{\zeta}=\tilde{\zeta}_{0}(u, \zeta)+O\left(\frac{1}{r}\right) .
$$

The stronger statement is as follows.

The stereographic coordinates of the sphere can be thought of as a map of the extended complex plane. In the asymptotic sphere defined by this setting, the angle transformation, at $u=$ const., must be conformal. But, a map of the extended complex plane onto itself is conformal if and only if it is a Moebius transformation; that is, a transformation of the form

$$
\tilde{\zeta}=\frac{a \zeta+b}{c \zeta+d}
$$

with $a b-b c \neq 0$.

We proceed in two stages.

First, note that the contravariant metric components for the standard Bondi like coordinate system is given by equations (3.13-18) of [6]; whose inverse is given by equations (3.19-24) of the same reference. The only difference is that for the general tetrad here considered

$$
g^{u r}=\frac{1}{g_{u r}}
$$

with $g_{u r}$ not necessarily being equal to 1 . In order to deduce the required relations we start from the null tetrad defined by (19),

$$
\left(\ell^{a}\right)=\left(A \frac{\partial}{\partial r}\right)^{a}
$$

(20) and (22). Then the contravariant components of the metric (the inverse metric), is given by

$$
\begin{aligned}
g^{u u} & =0, \\
g^{u r} & =A, \\
g^{u i} & =0, \\
g^{r r} & =2 U, \\
g^{r i} & =X^{i}, \\
g^{i j} & =-\left(\xi^{i} \bar{\xi}^{j}+\bar{\xi}^{i} \xi^{j}\right) .
\end{aligned}
$$

Then the metric is given by

$$
\begin{aligned}
g_{u r} & =\frac{1}{A} \\
g_{r r} & =0 \\
g_{r i} & =0 \\
g_{u u} & =-2 \frac{U}{A}+X^{i} X^{j} g_{i j}, \\
g_{u i} & =-g_{i j} X^{j} \\
g_{i j} & =\left(g^{i j}\right)^{-1}=-d \epsilon_{i k} \epsilon_{j l}\left(\xi^{i} \bar{\xi}^{j}+\bar{\xi}^{i} \xi^{j}\right) ;
\end{aligned}
$$

with $i, j, k, l=2,3, d=\operatorname{det}\left(g_{i j}\right), \epsilon_{i j}=-\epsilon_{j i}$ and $\epsilon_{23}=1$. In particular, defining the quantity

$$
\lambda=\epsilon_{i j} \xi^{i} \bar{\xi}^{j}
$$

one has that

$$
d=\frac{1}{|\lambda|^{2}}
$$

We will be interested in calculating the transformation of the radiation field component $\Psi_{4}^{0}$; which depends on the transformation properties of the dyad spinor $\iota^{A}$. This can be read-off from the transformation properties of the tetrad vector $n$. A straightforward calculation reveals that the one form $n_{a}$ is

$$
\begin{aligned}
\left(n_{a}\right)= & \left(g_{a b} n^{b}\right)=g_{u r} d r+g_{u u} d u+g_{u i} d x^{i} \\
& \quad+U g_{r u} d u+X^{i} g_{i u} d u+X^{i} g_{i j} d x^{j} \\
= & \frac{1}{A} d r \\
+ & \left(-2 \frac{U}{A}+X^{i} X^{j} g_{i j}+U \frac{1}{A}+X^{i}\left(-g_{i j} X^{j}\right)\right) d u \\
+ & \left(-g_{i j} X^{j}+X^{j} g_{i j}\right) d x^{i} \\
= & \frac{1}{A}(d r-U d u) .
\end{aligned}
$$

In the Bondi frame the following relations are satisfied,

$$
\begin{aligned}
\left(\tilde{\ell}_{a}\right) & =d \tilde{u} \\
\left(\tilde{m}_{a}\right) & =\frac{1}{\tilde{\lambda}} \epsilon_{i j} \tilde{\xi}^{i} \tilde{X}^{j} d \tilde{u}-\frac{1}{\tilde{\lambda}} \epsilon_{i j} \tilde{\xi}^{i} d \tilde{x}^{j}, \\
\left(\tilde{n}_{a}\right) & =d \tilde{r}-\tilde{U} d \tilde{u} .
\end{aligned}
$$


Additionally, in terms of the original coordinate system, the one-forms associated to the Bondi coordinates, to leading order, are given by

$$
\begin{gathered}
d \tilde{u}=\dot{\alpha} d u+\alpha_{\zeta} d \zeta+\alpha_{\bar{\zeta}} d \bar{\zeta}+O\left(\frac{1}{r}\right) \\
d \tilde{r}=\frac{1}{w} d r-\frac{r}{w^{2}}\left(\dot{w} d u+w_{\zeta} d \zeta+w_{\bar{\zeta}} d \bar{\zeta}\right)+O\left(r^{0}\right),
\end{gathered}
$$

and

$$
d \tilde{\zeta}=\dot{\tilde{\zeta}} d u+\tilde{\zeta}_{\zeta} d \zeta+\tilde{\zeta}_{\bar{\zeta}} d \bar{\zeta}+O\left(\frac{1}{r}\right) .
$$

Therefore from the contribution to the metric of $\tilde{\ell}_{a} \tilde{n}_{b}$, one deduces that

$$
\dot{\alpha} \frac{1}{w}=g_{u r}^{0} ;
$$

that is $w$ is determined by the choice of $\alpha$ and the value of $g_{u r}^{0}$.

In order to study the asymptotic transformation of the spinors $\tilde{\iota}^{A}$, we examine first the tetrad one-form $\tilde{n}_{a}$.

$$
\begin{aligned}
d \tilde{r}-\tilde{U} d \tilde{u}= & \frac{1}{w} d r-\frac{r}{w^{2}}\left(\dot{w} d u+w_{\zeta} d \zeta+w_{\bar{\zeta}} d \bar{\zeta}\right)+O\left(r^{0}\right) \\
& -\tilde{U}\left(\dot{\alpha} d u+\alpha_{\zeta} d \zeta+\alpha_{\bar{\zeta}} d \bar{\zeta}+O\left(\frac{1}{r}\right)\right) \\
= & \frac{1}{w}\left(d r-\left(\frac{r}{w} \dot{w}+\tilde{U} \dot{\alpha}\right) d u\right) \\
& +\frac{r}{w^{2}}\left(w_{\zeta} d \zeta+w_{\bar{\zeta}} d \bar{\zeta}\right)+(\text { lower orders }) .
\end{aligned}
$$

It is important to note that in the general case, the vector $n^{a}$, in the asymptotic region, will have a non zero component in the direction of the spacelike direction given by the conformal Bondi vectors $\hat{\tilde{m}}$ and $\hat{\tilde{\tilde{m}}}$. This can be seen from the fact that regular tetrad (with hat) at future null infinity is related to the standard tetrad in the asymptotic region by

$$
\begin{aligned}
\hat{\ell}_{a} & =\ell_{a}, \\
\hat{m}_{a} & =\Omega m_{a}, \\
\hat{n}_{a} & =\Omega^{2} n_{a},
\end{aligned}
$$

and

$$
\begin{aligned}
\hat{\ell}^{a} & =\Omega^{-2} \ell^{a}, \\
\hat{m}^{a} & =\Omega^{-1} m^{a}, \\
\hat{n}^{a} & =n^{a} ;
\end{aligned}
$$

where $\Omega$ is the conformal factor that defines the regular metric $\hat{g}_{a b}=\Omega^{2} g_{a b}$. Then, since in the asymptotic region

$$
\left(\tilde{m}_{a}\right)=\frac{\tilde{r}}{\sqrt{2} \tilde{P}_{0} \tilde{V}} d \overline{\tilde{\zeta}}+O\left(\tilde{r}^{0}\right),
$$

one can deduce

$$
\hat{n}^{a}\left(\hat{\tilde{m}}_{a}\right)=\frac{1}{\sqrt{2} \tilde{P}_{0} \tilde{V}}\left(\dot{\tilde{\tilde{\zeta}}}+X^{\bar{\zeta}} \overline{\tilde{\zeta}}_{\bar{\zeta}}\right)+O\left(\tilde{r}^{-1}\right) ;
$$

where $\Omega=\frac{1}{\tilde{r}}$ in this case. It is important here to realize the following. Extending the hypersurface $u=$ const. in the asymptotic region, one defines a section $S$ of future null infinity; in which by construction the vectors $\Omega^{-1} m^{a}$ and $\Omega^{-1} \bar{m}^{a}$ are tangent. Also, the regular extension of $\ell$, namely $\Omega^{-2} \ell^{a}$, is orthogonal to $S$. But, since future null infinity is a null hypersurface, one has that $n^{a}$, which is a null vector orthogonal to $S$, must be tangent to future null infinity. Since that regular extension of the Bondi frame to future null infinity is such that $\Omega^{-1} \tilde{m}^{a}$ and $\Omega^{-1} \overline{\tilde{m}}^{a}$ will be tangent to $S$; one deduces that the function $\zeta_{0}$ must be chosen such that

$$
\dot{\overline{\tilde{\zeta}}}+X^{\bar{\zeta}} \overline{\tilde{\zeta}}_{\bar{\zeta}}=0
$$

since the vector $\hat{\tilde{m}}_{a}$ must be orthogonal to $n^{a}$ at future null infinity.

Next, let us study the asymptotic form of $\tilde{n}^{a}$. Let us first note that

$$
\frac{\partial}{\partial \tilde{u}}=\frac{\partial u}{\partial \tilde{u}} \frac{\partial}{\partial u}+\frac{\partial \zeta}{\partial \tilde{u}} \frac{\partial}{\partial \zeta}+\frac{\partial \bar{\zeta}}{\partial \tilde{u}} \frac{\partial}{\partial \bar{\zeta}} ;
$$

where the last two terms are different from zero, since one has

$$
0=\frac{\partial \tilde{\zeta}}{\partial \tilde{u}}=\frac{\partial u}{\partial \tilde{u}} \frac{\partial \tilde{\zeta}}{\partial u}+\frac{\partial \zeta}{\partial \tilde{u}} \frac{\partial \tilde{\zeta}}{\partial \zeta} .
$$

Applying (89) onto $\tilde{u}$ and using (90) one can see that

$$
\frac{\partial u}{\partial \tilde{u}}=\frac{1}{\dot{\alpha}-\alpha_{\zeta} \frac{\dot{\tilde{\zeta}}_{0}}{\bar{\zeta}_{0 \zeta}}-\alpha_{\bar{\zeta}} \frac{\dot{\tilde{\zeta}}_{0}}{\tilde{\zeta}_{0 \bar{\zeta}}}},
$$

and

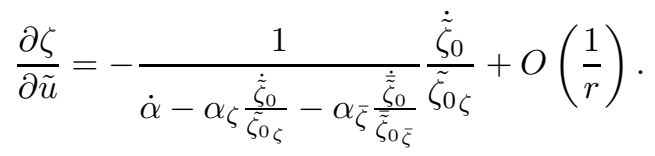

Similarly. one has

$$
\frac{\partial \bar{\zeta}}{\partial \tilde{u}}=-\frac{1}{\dot{\alpha}-\alpha_{\zeta} \frac{\dot{\tilde{\zeta}}_{0}}{\dot{\zeta}_{0 \zeta}}-\alpha_{\bar{\zeta}} \frac{\dot{\tilde{\tilde{\zeta}}}_{\tilde{\zeta}_{0 \bar{\zeta}}}}{\overline{\tilde{\zeta}}_{0 \bar{\zeta}}}}+O\left(\frac{1}{r}\right) ;
$$

and thus,

$$
\frac{\partial}{\partial \tilde{u}}=\frac{1}{\dot{\alpha}-\alpha_{\zeta} \frac{\dot{\tilde{\zeta}}_{0 \zeta}}{\tilde{\zeta}_{0 \zeta}}-\alpha_{\bar{\zeta}} \dot{\tilde{\zeta}}_{\tilde{\zeta}_{0 \bar{\zeta}}}}\left(\frac{\partial}{\partial u}-\frac{\dot{\tilde{\zeta}}}{\tilde{\zeta}_{\zeta}} \frac{\partial}{\partial \zeta}-\frac{\dot{\tilde{\zeta}}}{\tilde{\tilde{\zeta}}_{\zeta}} \frac{\partial}{\partial \bar{\zeta}}\right)
$$


To leading orders in the asymptotic tetrad transformation, $\tilde{n}$ and $n$ are related by

$$
\begin{aligned}
& \tilde{n}=\frac{\partial}{\partial \tilde{u}}+O\left(\frac{1}{r}\right) \\
& =\frac{1}{\dot{\alpha}-\alpha_{\zeta} \frac{\dot{\tilde{\zeta}}_{0}}{\tilde{\zeta}_{0 \zeta}}-\alpha_{\bar{\zeta}} \frac{\dot{\tilde{\zeta}}_{0}}{\tilde{\zeta}_{0 \bar{\zeta}}}}\left(\frac{\partial}{\partial u}-\frac{\dot{\tilde{\zeta}}}{\tilde{\zeta}_{\zeta}} \frac{\partial}{\partial \zeta}-\frac{\dot{\tilde{\zeta}}}{\overline{\tilde{\zeta}}_{\zeta}} \frac{\partial}{\partial \bar{\zeta}}\right)+O\left(\frac{1}{r}\right) \\
& =\frac{1}{\dot{\alpha}-\alpha_{\zeta} \frac{\dot{\tilde{\zeta}}_{0}}{\bar{\zeta}_{0 \zeta}}-\alpha_{\bar{\zeta}} \frac{\dot{\bar{\zeta}}}{\tilde{\zeta}_{0 \bar{\zeta}}}}\left(n-X_{0}^{\zeta} \frac{\partial}{\partial \zeta}-X_{0}^{\bar{\zeta}} \frac{\partial}{\partial \bar{\zeta}}\right. \\
& \left.-\frac{\dot{\tilde{\zeta}}}{\tilde{\zeta}_{\zeta}} \frac{\partial}{\partial \zeta}-\frac{\dot{\tilde{\zeta}}}{\overline{\tilde{\zeta}}_{\zeta}} \frac{\partial}{\partial \bar{\zeta}}\right)+O\left(\frac{1}{r}\right) \\
& =\frac{1}{\dot{\alpha}-\alpha_{\zeta} \frac{\dot{\tilde{\zeta}}_{0}}{\bar{\zeta}_{0 \zeta}}-\alpha_{\bar{\zeta}} \frac{\dot{\bar{\zeta}}_{0}}{\tilde{\zeta}_{0 \bar{\zeta}}}} n+O\left(\frac{1}{r}\right) \text {, }
\end{aligned}
$$

since by virtue of (88) and its complex conjugate, the asymptotic terms involving spatial directions cancel.

Additionally,

$$
0=\frac{\partial \tilde{u}}{\partial \tilde{\zeta}}=\frac{\partial u}{\partial \tilde{\zeta}} \frac{\partial \tilde{u}}{\partial u}+\frac{\partial \zeta}{\partial \tilde{\zeta}} \frac{\partial \tilde{u}}{\partial \zeta}=\dot{\alpha} \frac{\partial u}{\partial \tilde{\zeta}}+\alpha_{\zeta} \frac{\partial \zeta}{\partial \tilde{\zeta}} ;
$$

thus

$$
\frac{\partial \zeta}{\partial \tilde{\zeta}}=-\frac{\dot{\alpha}}{\alpha_{\zeta}} \frac{\partial u}{\partial \tilde{\zeta}}
$$

Last, from

$1=\frac{\partial \tilde{\zeta}}{\partial \tilde{\zeta}}=\frac{\partial u}{\partial \tilde{\zeta}} \frac{\partial \tilde{\zeta}}{\partial u}+\frac{\partial \zeta}{\partial \tilde{\zeta}} \frac{\partial \tilde{\zeta}}{\partial \zeta}=\dot{\tilde{\zeta}} \frac{\partial u}{\partial \tilde{\zeta}}+\tilde{\zeta}_{\zeta} \frac{\partial \zeta}{\partial \tilde{\zeta}}=\dot{\tilde{\zeta}} \frac{\partial u}{\partial \tilde{\zeta}}-\tilde{\zeta}_{\zeta} \frac{\dot{\alpha}}{\alpha_{\zeta}} \frac{\partial u}{\partial \tilde{\zeta}}$

one has

$$
\frac{\partial u}{\partial \tilde{\zeta}}=\frac{\alpha_{\zeta}}{\alpha_{\zeta} \dot{\tilde{\zeta}}-\dot{\alpha} \tilde{\zeta}_{\zeta}}
$$

and

$$
\frac{\partial \zeta}{\partial \tilde{\zeta}}=-\frac{\dot{\alpha}}{\alpha_{\zeta} \dot{\tilde{\zeta}}-\dot{\alpha} \tilde{\zeta}_{\zeta}}
$$

The asymptotic behavior of the vector $\tilde{m}$ is therefore

$$
\begin{aligned}
& \tilde{m}= \frac{\sqrt{2} \tilde{P}}{\tilde{r}} \frac{\partial}{\partial \tilde{\zeta}}+O\left(\frac{1}{r^{2}}\right) \\
&= \frac{\sqrt{2} P_{0} \tilde{V} w}{r}\left(-\frac{\alpha_{\zeta}}{\dot{\alpha} \tilde{\zeta}_{\zeta}-\alpha_{\zeta} \dot{\tilde{\zeta}}} \frac{\partial}{\partial u}+\frac{\dot{\alpha}}{\dot{\alpha} \tilde{\zeta}_{\zeta}-\alpha_{\zeta} \dot{\tilde{\zeta}}} \frac{\partial}{\partial \zeta}\right) \\
& \quad O\left(\frac{1}{r^{2}}\right) \\
&=-\frac{\sqrt{2} P_{0} \tilde{V} w}{r} \frac{\alpha_{\zeta}}{\dot{\alpha} \tilde{\zeta}_{\zeta}-\alpha_{\zeta} \dot{\zeta}} n+\frac{\dot{V} w}{V} \frac{\dot{\alpha}}{\dot{\alpha} \tilde{\zeta}_{\zeta}-\alpha_{\zeta} \dot{\zeta}} m \\
& \quad+O\left(\frac{1}{r^{2}}\right) .
\end{aligned}
$$

Since the metric expressed in terms of the new null tetrad must coincide with the metric expressed in terms of the original null tetrad, it is deduced that

$$
1=\tilde{V}=\frac{V\left|\dot{\alpha} \tilde{\zeta}_{\zeta}-\alpha_{\zeta} \dot{\tilde{\zeta}}\right|}{w \dot{\alpha}}=\frac{V g_{u r}^{0}\left|\dot{\alpha} \tilde{\zeta}_{\zeta}-\alpha_{\zeta} \dot{\tilde{\zeta}}\right|}{\dot{\alpha}^{2}}
$$

where we have used that the tilde system is Bondi like.

One then can deduce the transformation

$$
\tilde{\iota}^{A}=\frac{1}{\sqrt{\dot{\alpha}-\alpha_{\zeta} \frac{\dot{\tilde{\zeta}}_{0}}{\bar{\zeta}_{0 \zeta}}-\alpha_{\bar{\zeta}} \frac{\dot{\bar{\zeta}}_{0}}{\tilde{\zeta}_{0 \bar{\zeta}}}}} \iota^{A} .
$$

Consequently the radiation component $\Psi_{4}^{0}$ transforms in the following way

$$
\begin{aligned}
\tilde{\Psi}_{4}^{0}= & \tilde{\Omega}^{-1} \Psi_{A B C D} \hat{\tilde{\iota}}^{A} \hat{\tilde{\iota}}^{B} \hat{\tilde{\iota}}^{C} \hat{\tilde{\iota}}^{D} \\
= & \frac{1}{w} \frac{1}{\left(\dot{\alpha}-\alpha_{\zeta} \frac{\dot{\tilde{\zeta}}_{0}}{\bar{\zeta}_{0 \zeta}}-\alpha_{\bar{\zeta}} \frac{\dot{\tilde{\zeta}}_{0}}{\tilde{\zeta}_{0 \bar{\zeta}}}\right)^{2}} \Psi_{4}^{0} \\
= & \frac{g_{u r}^{0}}{\dot{\alpha}\left(\dot{\alpha}-\alpha_{\zeta} \frac{\dot{\tilde{\zeta}}_{0}}{\tilde{\zeta}_{0 \zeta}}-\alpha_{\bar{\zeta}} \frac{\dot{\tilde{\zeta}}_{0}}{\tilde{\zeta}_{0 \bar{\zeta}}}\right)^{2}} \Psi_{4}^{0} .
\end{aligned}
$$

Equivalently, this can be expressed as

$$
\tilde{\Psi}_{4}^{0}=\frac{g_{u r}^{0}}{\dot{\alpha}\left(\dot{\alpha}+\alpha_{\zeta} X_{0}^{\zeta}+\alpha_{\bar{\zeta}} X_{0}^{\bar{\zeta}}\right)^{2}} \Psi_{4}^{0}
$$

Let us emphasize that $\tilde{\zeta}_{0}$ is chosen to make $\tilde{X}_{0}=0$; while $\alpha$ is chosen to make $V=1$.

This complicated transformation of the radiation field indicates the convenience of adapting the numerical code such that $X^{i}=0+O\left(r^{-1}\right)$; so that $\dot{\tilde{\zeta}}=0$. Otherwise the determination of $\alpha$, needed in (104) or (105), would be very difficult.

\section{EXAMPLES}

\section{A. Conventions and formulae. Factors of 2}

In section I we presented the standard expressions for the radiated four-momentum. Yet, there are differences in factors with the definitions employed in numerical implementations. The difference arises from the frame adopted for the extraction procedure. The analysis presented in this work can be used to clarify these differences. In a Bondi frame, the expression for the radiated four-momentum $P^{a}$ is,

$$
\dot{P}^{a}=-\frac{1}{4 \pi} \int_{S} \hat{l}^{a} \dot{\sigma}^{0} \dot{\bar{\sigma}}^{0} d S^{2} .
$$

(with denoting a derivative with respect to $\tilde{u}$ ) This, can be re-expressed in terms of $\Psi_{4}^{0}$ by employing the identity 
valid in a Bondi frame $\tilde{\Psi}_{4}^{0}=-\ddot{\bar{\sigma}}^{0}$. Where we have used the ${ }^{\sim}$ to denote a quantity obtained in a Bondi frame (we don't do that for $\sigma^{0}$ as here we only use it as obtained in the Bondi frame).

$$
\dot{P}^{a}=-\frac{1}{4 \pi} \int_{S} \hat{l}^{a}\left|\int_{-\infty}^{\tilde{u}} \tilde{\Psi}_{4}^{0} d \tilde{u}^{\prime}\right|^{2} d S^{2} .
$$

In a numerical implementation, as discussed, the readily available $\Psi_{4}^{0}$ differs from the Bondi one by

$$
\tilde{\Psi}_{4}=\frac{\Psi_{4}}{\left(g_{u r}^{0}\right)^{2} V^{3}} .
$$

Thus

$$
\dot{P}^{a}=-\frac{1}{4 \pi} \int_{S} \hat{l}^{a}\left|\int_{-\infty}^{\tilde{u}} \frac{\Psi_{4}}{\left(g_{u r}^{0}\right)^{2} V^{3}} d \tilde{u}^{\prime}\right|^{2} d S^{2} .
$$

1. Standard approach, boost ambiguity and resulting factors of 2

We recall that the null tetrads are not uniquely defined as they have the boost freedom $\ell^{a} \rightarrow \ell^{a} \lambda^{-1}, n^{a} \rightarrow n^{a} \lambda$ and spin freedom $m^{a} \rightarrow m^{a} e^{i \gamma}[21]$. This freedom can bring about additional factors in the resulting expression for the Weyl scalars, fortunately a Bondi frame naturally fixes this ambiguity. In what follows we illustrate how this freedom is fixed by ensuring a Bondi tetrad frame is adopted in a simple exmaple. This, in passing, will make evident the different factors encountered in commonly employed formulae. In the extraction procedure it has become custommary to introduce a tetrad frame in the following form [42]. First an extraction worldtube is defined as the Cartesian $x^{2}+y^{2}+z^{2}=R^{2}$. Then, three vectors (labeled by $J$ ) $\tilde{v}_{J}^{i}$ at a hypersurface slice are adopted in the following way:

$\tilde{v}_{1}^{i}=(-y, x, 0), \tilde{v}_{2}^{i}=(x, y, z)^{i}, \tilde{v}_{3}^{i}=\operatorname{det}(\gamma)^{(1 / 2)} \gamma^{i j} \epsilon_{j l m} v_{1}^{l} v_{2}^{m} ;$

where $\gamma_{i j}$ is the induced metric on the spacelike hypersurface at a given time. In our present case, this reduces to

$$
\tilde{v}_{1}^{i}=\partial_{\phi}^{i}, \tilde{v}_{2}^{i}=\partial_{r}^{i}, \tilde{v}_{3}^{i}=\partial_{\theta}^{i} .
$$

The next step involves a Gram-Schmidt procedure to construct three orthonormal vectors $v_{J}^{i}$ with respect to $\gamma_{i j}$. Finally four spacetime vectors are easily constructed by $r^{a}=\left(0, v_{2}\right) ; \theta^{a}=\left(0, v_{3}\right) ; \phi^{a}=\left(0, v_{1}\right)$, which together with $N^{a}$ (the unit timelike vector normal to the hypersurface) can be employed to construct the tetrad as,

$$
\begin{aligned}
\ell^{\prime a} & =\frac{1}{\sqrt{2}}\left(N^{a}+r^{a}\right) \\
n^{\prime a} & =\frac{1}{\sqrt{2}}\left(N^{a}-r^{a}\right) \\
m^{\prime a} & =\frac{1}{\sqrt{2}}\left(\theta^{a}+i \phi^{a}\right),
\end{aligned}
$$

with $r^{2}=x^{2}+y^{2}+z^{2}$. Consider the simplest case of a spacetime whose metric, is given by $g_{a b}=\eta_{a b}+h_{a b}$ with $h_{a b}$ a sufficiently fast decaying functions at far distances and $\eta_{a b}$ a flat metric in Cartesian coordinates. In this case, to leading order, the tetrad resulting from the standard procedure is,

$$
\begin{aligned}
\ell^{\prime a} & =\frac{1}{\sqrt{2}}\left(\partial_{t}^{a}+\partial_{R}^{a}\right), \\
n^{\prime a} & =\frac{1}{\sqrt{2}}\left(\partial_{t}^{a}-\partial_{R}^{a}\right), \\
m^{\prime a} & =\frac{1}{r \sqrt{2}}\left(\partial_{\theta}^{a}+\frac{i}{\sin (\theta)} \partial_{\phi}^{a}\right) .
\end{aligned}
$$

The main difference with the previous tetrads is that in the former one has used a null coordinate $u$ that in this case would be of the form $u=t-R$; with the first null tetrad vector given by $\ell_{a}=d u_{a}$. Therefore, one has $\ell^{\prime a}=\frac{1}{\sqrt{2}} \ell^{a}$ in this case and so $\iota^{\prime A}=2^{\frac{1}{4}} \iota^{A}$. Then, in the calculation of the radiation component of the Weyl tensor one would have $\Psi_{4}^{\prime}=2 \Psi_{4}$. The expression for the radiated momentum in these coordinates results,

$$
\dot{P}^{a}=-\frac{1}{16 \pi} \int_{S} \hat{l}^{a}\left|\int_{-\infty}^{u} \Psi_{4}^{\prime} d \tilde{u}\right|^{2} d S^{2} .
$$

This then explains the factor of 4 difference in the expressions cited in 25, 28, 29] with that in eqn (12) (and those in the standard literature [16, 24], etc.).

\section{B. Teukolsky waves and wave extraction}

As a second example, we adopt linearized waves on flat spacetime as given by the so called Teukolsky waves 43]. We adopt this example, coupled with a possible coordinate transformation to illustrate the differences that may arise when suitable contact with a Bondi frame is missing. We adopt the simplest expression for the line element given by

$$
\begin{aligned}
d s^{2}= & d t^{2}-\left(1+A f_{r r}\right) d r^{2}-2 B f_{r \theta} d r d \theta \\
& -\left(1+3 C f_{\theta \theta}^{1}-A\right) r^{2} d \theta^{2} \\
& -\left(1+3 C f_{\phi \phi}^{1}-A f_{\phi \phi}^{2}\right) r^{2} \sin (\theta)^{2} d \phi^{2}
\end{aligned}
$$

where

$$
\begin{aligned}
f_{r r}=2-3 \sin (\theta)^{2} ; \quad f_{r \theta} & =-3 \sin (\theta) \cos (\theta) \\
f_{\theta \theta}^{1}=-f_{\phi \phi}^{1}=f_{\phi \phi}^{2}+1 & =3 \sin (\theta)^{2} .
\end{aligned}
$$

and

$$
\begin{gathered}
A=3\left(\frac{F^{(2)}}{r^{3}}+\frac{3 F^{(1)}}{r^{4}}+\frac{3 F}{r^{5}}\right) \\
B=-\left(\frac{F^{(3)}}{r^{2}}+\frac{3 F^{(2)}}{r^{3}}+\frac{6 F^{(1)}}{r^{4}}+\frac{6 F}{r^{5}}\right) \\
C=\frac{1}{4}\left(\frac{F^{(4)}}{r}+\frac{2 F^{(3)}}{r^{2}}+\frac{9 F^{(2)}}{r^{3}}\right. \\
\left.+\frac{21 F^{(1)}}{r^{4}}+\frac{21 F}{r^{5}}\right)
\end{gathered}
$$


with $F=F(t-r), F^{(n)}=\left(\frac{d^{n} F(x)}{d x^{n}}\right)_{x=t-r}$.

The Riemann tensor for such a line element can be straightforwardly constructed. Since to leading order the line element is just the flat metric, it is straightforward to identify the Bondi frame. A simple calculation gives,

$$
\tilde{\Psi}_{4}^{0}=\frac{3}{8} \sin (\theta)^{2} F^{(6)}(t-r)
$$

On the other hand, we can calculate $\Psi_{4}$ with the standard procedure. We consider two cases: (I) calculating $\Psi_{4}$ in the coordinates $(t, r, \theta, \phi)$ and (II) considering the transformation $\tilde{r} \rightarrow r g(t)$ which induces a non-trivial $V(t)$. The induced tetrad, to the order that enters in the calculation of the leading order of $\Psi_{4}$ are

$$
\begin{aligned}
\ell^{a} & =\frac{1}{\sqrt{2}}\left(\partial_{t}^{a}+\frac{1}{g}(1-r \dot{g}) \partial_{R}^{a}\right), \\
n^{a} & =\frac{1}{\sqrt{2}}\left(\partial_{t}^{a}-\frac{1}{g}(1-r \dot{g}) \partial_{R}^{a}\right), \\
m^{a} & =\frac{1}{r g \sqrt{2}}\left(\partial_{\theta}^{a}+i \partial_{\phi}^{a}\right) .
\end{aligned}
$$

Taking $g \equiv 1$ gives the tetrad for case (I). A straightforward calculation gives this case,

$$
\Psi_{4}^{0}=\frac{3}{4} F^{(6)}(t-r) \sin (\theta)^{2}
$$

while for case (II)

$$
\Psi_{4}^{0}=\frac{3}{4} \frac{F^{(6)}(t-r g) \sin (\theta)^{2}}{g} .
$$

Notice a difference of a factor $g^{-1}$ appearing in case (II) in addition to a factor of 2 when compared to eqn (119). These factors result from $V=g^{-1}$ and $g_{u r}^{0}=\sqrt{2} g$. The corrections described in section IV can be used to reconcile these differences and obtain Bondi's expression in a way mostly independent of gauge issues. In the next section we discuss how the different factors involved can be obtained in generic settings.

\section{OBTAINING THE MISSING LINKS}

From our previous discussion, it is clear that one must take into account the effects caused by the conformal factor $V$ and the induced metric components $g_{u r}, g_{u i}$. These later factors can be read-off from the expression of the null one-form $\tilde{n}_{a}$ in the induced null coordinates at $\Gamma$. Namely $l_{a}=d u_{a}$ defines the function $u$ and the transformation from the $(t, r, \zeta, \bar{\zeta})$ to the $(u, r, \zeta, \bar{\zeta})$ coordinates can be employed to express $n_{a}$ in this system. Then, $g_{u r}^{-1}=l^{a} d r_{a} ; X^{i}=n^{a} d x_{a}^{i}$.

The extraction of the conformal factor $V$ involves more work but can be obtained by considering a conformal transformation of the sphere and evaluating its curvature scalar 44]. Recall that any metric on $S^{2}$ is conformally related to that of the unit sphere, thus it must be the case that $\hat{g}_{i j} \equiv g_{i j} R^{-2}=\omega^{-2} q_{i j}$. Thus, the unit sphere metric and the induced metric at the worldtube at a constant time are related by a conformal factor $\omega$. With our conventions then $V=\omega$. A way to solve for this factor is to compute the scalar curvature of the cut $S$ on the worldtube. This gives rise to the following relation

$$
\mathcal{R}=2\left(V^{2}+D^{A} D_{A} \ln V\right),
$$

where $\mathcal{R}$ is the Ricci scalar curvature and $D_{i}$ is the covariant derivative of the metric $\hat{g}_{i j}$. Let us observe that in two dimensions one has $\mathcal{R}=2 K_{V}$.

This equation can be solved to obtain $V$. Notice that this equation admits more than one solution due to the rotational group of transformations. One way to solve it, and implicitly fix this freedom is motivated in techniques introduced to find apparent horizons in numerical simulation [45, 46]. In this approach we express $V$ (or a related quantity) in terms of a spherical-harmonic expansion, and then obtain a recursive relation to solve for the expansion coefficients. We begin by considering [51]

$$
W \equiv V^{2}=\Sigma_{l m} a_{l m} Y_{l m}
$$

with $Y_{l m}$ spherical harmonics satisfying $\bar{\nabla}^{2} Y_{l m}=-l(l+$ 1) $Y_{l m}$ with $\bar{\nabla}^{2}$ the Laplacian operator with respect to the unit sphere metric expressed in the same coordinates as those where $\hat{g}_{i j}$ is known (we will refer to this as $\bar{q}_{i j}$ ). The solution we seek satisfies $H(W)=0$ with

$$
H=2 W+D^{i} D_{i} \ln W-\mathcal{R} .
$$

Next, we consider the equation

$$
\rho H(W)+\bar{\nabla}^{2} W=\bar{\nabla}^{2} W
$$

with $\rho$ a function to be determined in a suitable manner. The solution we seek turns the equation above into an identity. Consider now integrating this equation over the sphere having replaced our anzats for $W$. This will provide a recursion relation for obtaining the parameters $a_{l m}($ for $l \neq 0)$ as,

$$
\int_{S} \bar{Y}_{l m}\left(\rho H+\bar{\nabla}^{2} W\right) d \Omega=-l(l+1) a_{l m}
$$

Thus, starting with trial values for $\left\{a_{l m}\right\}$ a new set can be obtained through the equation above. Two ingredients remain to be provided however, one is how $a_{00}$ is to be set and the other a plausibility argument for the convergence of the method. We describe first the latter issue and then discuss the former. This argument relies on concentrating on the principal part of the system $\left(\rho H+\bar{\nabla}^{2} W\right)$ and to exploit $\rho$ to our advantage. Inspection of these terms allows one to re-express them as $M^{i j} \bar{D}_{i} \bar{D}_{j} W+S$ with

$$
\begin{aligned}
M^{i j}= & \frac{\rho}{W} \hat{g}^{i j}+\bar{q}^{i j} \\
S= & \rho\left(2 W-\frac{1}{W} \hat{g}^{i j} C_{i j}^{k} \bar{D}_{k} W\right. \\
& \left.-\frac{1}{W^{2}} \hat{g}^{i j} \bar{D}_{i} W \bar{D}_{j} W-\mathcal{R}\right) .
\end{aligned}
$$


with $\bar{D}$ the covariant derivative associated with $\bar{q}_{i j}$ and $C_{i j}^{k}$ the tensor relating the connections $\bar{D}_{i}$ with $D_{i}$. Notice now that equation (125) can be formally solved by the iteration scheme

$$
W^{(n+1)}=\left(\bar{\nabla}^{2}\right)^{-1}\left(\rho H+\bar{\nabla}^{2}\right) W^{(n)}
$$

Here the standard argument applies, the Laplacian operator smoothes out high frequencies and by properly adjusting $M^{i j}$ such that the highest order derivatives are removed the successive solutions will be smoother which is a requirement for convergence. The factor $\rho$ can be chosen so as to cancel particular terms in the secondorder derivatives, or try to cancel as much as possible $M^{i j}$ (see the discussion in [45]. For instance, if $\bar{q}^{i j} \simeq q^{i j}$ the trivial choice $\rho=-1$ is ideally suited for this purpose.

Last we comment on how $a_{00}$ is obtained. At the first step of the iteration, a useful choice is induced by assuming $W^{(1)}=a_{00}^{1} Y_{00}$. Then, one simply has

$$
0=\int\left(2 W^{(1)}-\mathcal{R}\right) Y_{00} d \Omega=2 a_{00}^{1}-\int \mathcal{R} Y_{00} d \Omega
$$

At subsequent steps, having fixed $a_{l m}^{n+1}$ for $l>0$, equation (126) can be employed to fix $a_{00}^{n+1}$. However, due to the non-linearities of the equation this might turn out to be difficult. A simpler, more direct route, is to do so from

$$
\int_{S}\left(\left(2 W^{(n+1)}\right)+D^{i} D_{i} \ln W^{(n)}-\mathcal{R}\right) Y_{00} d \Omega=0
$$

Which can be used to determine $a_{00}^{n+1}$ after all other coefficients have been obtained.

\section{FINAL COMMENTS}

In this work we have re-examined the issue of computing gravitational radiation effects through the use of Weyl scalars. The analysis reveals which correcting factors are to be accounted for if the coordinates adopted do not conform to a Bondi system. To date while these corrections have not been taken into account in numerical efforts the waveforms obtained both appear quite reasonable and, most importantly, agreeing across different implementations. What is then the expected contribution of the corrections indicated here or the relevance of the present discussion in light of these observations?

First, at least the formalism presented in this work allows for easily enforcing consistency among different implementations. Namely, while coordinates typically used vary among different efforts, considering the transformation to a Bondi system provides a common frame for the computation. As a result, comparison of obtained signals from different codes would be expedited. Second, the correcting terms will have generically non-trivial angular dependence. Therefore, the decomposition on different multipolar moments would be affected. In the case of non-spinning equal mass black holes symmetry considerations indicate this might not be a significant issue. On the other hand, for different masses and/or spinning compact objects the contribution should be non-trivial, especially in light of significant kicks observed which indicate a strong direction dependent of the radiated waves.

It is important to stress here that the corrections are case-by-case dependent as they are both sensitive to the gauge and initial data employed. Consequently it is difficult to assess the role the corrections indicated here might have. Nevertheless, consistency with the calculation formalism and a simplified frame of comparison among different implementations are already key reasons for the relevance of the discussion.

As a side comment we want to stress we have employed a convention based on a $(-2)$ signature following [21, 22] which is the standard signature employed in studies of asymptotically flat spacetimes.

Last, short of considering the corrections discussed in this work, as discussed in [47], one could estimate whether these effects may play a role by evaluating the norms $\|\mathcal{R}-2\|,\left\|g_{u r}-\kappa\right\|$ (with $\kappa=1$ or $\sqrt{2}$ depending on the boost freedom adopted for $\ell^{a}$ ) and $\left\|g_{u A}\right\|$. If these norms are at the order of the truncation error in a simulation, then the correcting factors would certainly not be essential. We will examine these issues for different binary systems in a forthcoming work [48].

\section{ACKNOWLEDGMENTS}

We would like to thank D. Garfinkle, C. Palenzuela, F. Pretorius, J. Pullin M. Tiglio and J. Winicour for comments and discussions. This work was supported in part by grants from NSF: PHY-0326311 and PHY-0554793 to Louisiana State University and ANPCyT, CONICET and SeCyT-UNC. L.L. wishes to thank the University of Cordoba for hospitality where parts of this work were completed.
[1] V. Moncrief. Ann. Phys. (NY), 88, 323 (1974).

[2] T. Regge and J.A. Wheeler, Phys. Rev. 108, 1063 (1957).
[3] F. Zerilli, Phys. Rev. Lett. 24, 737 (1970).

[4] S. Teukolsky. Astroph. Journal, 185, 635-647, (1973). 
[5] A. M. Abrahams and R. H. Price, Phys. Rev. D 53, 1963 (1996).

[6] Osvaldo M. Moreschi. Class. Quantum Grav., 4:10631084, 1987.

[7] E. Newman and R. Penrose, Journal of Math Phys, 3, 566-578, (1962).

[8] E. Newman and T. Unti, Journal of Math Phys, 3, 891901, (1962).

[9] M. Babiuc, B. Szilagyi, I. Hawke and Y. Zlochower, Class. Quant. Grav. 22, 5089 (2005)

[10] N. T. Bishop, R. Gomez, L. Lehner and J. Winicour, Phys. Rev. D 54, 6153 (1996).

[11] N. T. Bishop, R. Gomez, L. Lehner, B. Szilagyi, J. Winicour and R. A. Isaacson, in "On the black hole trail", Eds B. Iyer and B. Bhawal. Kluwer, (1998).

[12] H. Friedrich, Lect. Notes Phys. 604, 1 (2002) arXiv:gr-qc/0209018.

[13] H. Bondi, M. van der Burg and A. Metzner, Proc. R. Soc. London, Ser. A 269, 21 (1962).

[14] R. Sachs, Proc. R. Soc. London, Ser. A 270, 103 (1962).

[15] R. Penrose, Phys. Rev. Lett. 10, 66 (1963).

[16] E.T. Newamn and P. Tod. in "General Relativity and Gravitation", Ed. A. Held, Plenum Press, New York (1980).

[17] E. Newman and T. Unti, Journal of Math Phys, 6, 18061811, (1965).

[18] L.A. Tamburino and J. Winicour, Phys. Rev. D 150, 1039 (1966).

[19] J. Winicour, G.R.G. 19N3, 281, (1987).

[20] Osvaldo M. Moreschi. Class. Quantum Grav., 3:503-525, 1986.

[21] R. Geroch, A. Held, and R. Penrose. J. Math. Phys., 14:874-881, 1973.

[22] F. A. E. Pirani. Introduction to gravitational radiation theory. In Trautman, F. A. E. Pirani, and H. Bondi, editors, Brandeis Summer Institute in Theoretical Physics 1964, Volume One: Lectures on general relativity. Prentice-Hall Inc., 1965.

[23] Osvaldo M. Moreschi. Class. Quantum Grav., 5:423-435, 1988.

[24] L. Smarr, in "Sources of Gravitational Radiation" Ed. L. Smarr, Cambridge Univ. Press, New York (1979).

[25] J. G. Baker, M. Campanelli and C. O. Lousto, Phys. Rev. D 65, 044001 (2002)

[26] M. Campanelli, C. O. Lousto, Y. Zlochower, B. Krishnan and D. Merritt, Phys. Rev. D 75, 064030 (2007).

[27] F. Pretorius, Phys. Rev. Lett. 95, 121101 (2005)
[28] B. Bruegmann, J. A. Gonzalez, M. Hannam, S. Husa, U. Sperhake and W. Tichy, arXiv:gr-qc/0610128

[29] J. G. Baker, J. Centrella, D. I. Choi, M. Koppitz and J. van Meter, Phys. Rev. D 73, 104002 (2006) arXiv:gr-qc/0602026.

[30] C. Palenzuela, I. Olabarrieta, L. Lehner and S. Liebling, Phys. Rev. D 75, 064005 (2007)

[31] C. O. Lousto and Y. Zlochower, arXiv:gr-qc/0703061

[32] H. P. Pfeiffer, D. A. Brown, L. E. Kidder, L. Lindblom, G. Lovelace and M. A. Scheel, arXiv:gr-qc/0702106

[33] M. Koppitz, D. Pollney, C. Reisswig, L. Rezzolla, J. Thornburg, P. Diener and E. Schnetter, arXiv:gr-qc/0701163

[34] J. R. van Meter, J. G. Baker, M. Koppitz and D. I. Choi, Phys. Rev. D 73, 124011 (2006)

[35] F. Pretorius, Class. Quant. Grav. 23, S529 (2006)

[36] M. Alcubierre, B. Brugmann, P. Diener, M. Koppitz, D. Pollney, E. Seidel and R. Takahashi, Phys. Rev. D 67, 084023 (2003)

[37] W. Kinnersley, J. Math. Phys. 10, 1195 (1969).

[38] M. Campanelli, B. J. Kelly and C. O. Lousto, Phys. Rev. D 73, 064005 (2006)

[39] L. M. Burko, Phys. Rev. D 75, 084039 (2007).

[40] A. Nerozzi, M. Bruni, L. M. Burko and V. Re, AIP Conf. Proc. 861, 702 (2006) arXiv:gr-qc/0607066.

[41] E. Pazos, E. N. Dorband, A. Nagar, C. Palenzuela, E. Schnetter and M. Tiglio, arXiv:gr-qc/0612149.

[42] J. G. Baker, M. Campanelli and C. O. Lousto, Phys. Rev. D 65, 044001 (2002).

[43] S. Teukolsky. Phys. Rev. D, 26, 745-750, (1982).

[44] N. T. Bishop, R. Gomez, L. Lehner, M. Maharaj and J. Winicour, Phys. Rev. D 56, 6298 (1997)

[45] C. Gundlach, Phys. Rev. D 57, 863 (1998).

[46] T. Nakamura, K. Kojima and K. Oohara, Phys. Lett, 106A, 235 (1984).

[47] T. Baumgarte, P. Brady, J. D. E. Creighton, L. Lehner, F. Pretorius and R. DeVoe, arXiv:gr-qc/0612100

[48] C. Palenzuela, M. Anderson, et. al. In preparation (2007).

[49] For instance by employing the extracted values at different radii to determine the leading behavior of $\Psi_{4}$ by fitting a suitable polynomial in $R^{-1}$.

[50] By calculating $\Psi_{4}$ at different extraction radii, this radial behavior can be checked

[51] -Alternatively, one could have expressed $V$ itself in terms of a spectral series-. 